01

\title{
Моделирование процесса коррекции локальных ошибок формы поверхности малоразмерным ионным пучком
}

\author{
(C) А.К. Чернышев, ${ }^{1,2}$ И.В. Малышев, ${ }^{1}$ А.Е. Пестов, ${ }^{1}$ Н.И. Чхало ${ }^{1}$ \\ ${ }^{1}$ Институт ффизики микроструктур РАН, \\ 607680 Нижний Новгород, Россия \\ ${ }^{2}$ Нижегородский государственный университет им. Н.И. Лобачевского, \\ 603950 Нижний Новгород, Россия \\ e-mail: aepestov@ipm.sci-nnov.ru
}

Поступило в Редакцию 28 марта 2019 г.

В окончательной редакции 28 марта 2019 г.

Принято к публикации 15 апреля 2019 г.

\begin{abstract}
Предложен алгоритм решения задачи коррекции локальных ошибок формы поверхности малоразмерным ионным пучком. Алгоритм предполагает последовательный перебор возвышений относительно среднего с целью поиска наиболее оптимальной точки травления, удовлетворяющей критерию - уменьшение суммы модулей производных на пятне травления. Показано, что новый подход позволяет заметно расширить диапазон пространственных частот, поддающихся воздействию при заданном размере ионного пучка.
\end{abstract}

Ключевые слова: ЭУФ оптика, форма поверхности, ионно-пучковая коррекция формы, ионное травление.

DOI: 10.21883/JTF.2019.11.48323.133-19

\section{Введение}

В ИФМ РАН активно развиваются методы формирования прецизионных оптических элементов мягкого рентгеновского (МР) и экстремального ультрафиолетового (ЭУФ) диапазонов длин волн, обеспечивающих дифракционное качество изображения. Интерес к этим диапазонам обусловлен в первую очередь возможностью существенного повышения разрешения оптических систем по сравнению с видимым и ультрафиолетовым излучением. Флагманом в развитии является проекционная литография, с помощью которой в мире уже производятся микропроцессоры с рекордными топологическими параметрами. Значительный интерес также вызывает рентгеновская астрономия, изучающая процессы, протекающие на звездах, в частности, на Солнце. Новым направлением является рентгеновская микроскопия, призванная помочь медикам и биологам изучать живые объекты с пространственным разрешением на уровне $10-20 \mathrm{~nm}$ и проводить $z$-томографию [1].

Согласно критерию Рэлея (1), пространственное разрешение $(\delta x)$ определяется в первую очередь длиной волны излучения:

$$
\delta x=0.61 \cdot \lambda / N A,
$$

где $\lambda$ - рабочая длина волны света, $N A$ - числовая апертура объектива. Из критерия Рэлея следует, что чем короче длина волны, тем меньшие объекты могут быть разрешены. Однако он рассматривает идеальный случай, т. е. когда формирующие изображения линзы или зеркала идеальной, например, сферической формы. В случае, если это не так, дифракционный предел может быть достигнут, в случае выполнения критерия Марешаля

$$
\mathrm{RMS} \leq \lambda / 14
$$

Критерий Марешаля [2] гласит, что оптическая система будет обеспечивать пространственное разрешение в соответствие с дифракционным пределом Рэлея, если качество поверхностей (по параметру RMS среднеквадратическое отклонение) будет лучше, чем $\lambda / 14$. В этом случае разрешение происходит по уровню интенсивности 0.5 , что считается достаточным. Соответственно переход в МР и ЭУФ диапазонов длин волн дает возможности, с одной стороны, существенно повысить пространственное разрешение оптических систем вследствие существенного сокращения длины волны (1). А с другой стороны, короткая длина волны $(2.0-15 \mathrm{~nm})$ накладывает высокие требования на качество оптических поверхностей (2). И если учесть, что длина волны составляет единицы нанометров, то точность формы оптической поверхности должна быть на уровне десятых долей нанометра.

Задача формирования оптических поверхностей с точностью формы лучше $1.0 \mathrm{~nm}$ по параметру среднеквадратического отклонения решается при помощи коррекции локальных ошибок формы травлением малоразмерным пучком ускоренных ионов при движении по заданной траектории [3-5]. В литературе имеется большое количество работ, описывающих процедуру обработки поверхности сканированием пучком с гауссовым распределением плотности ионного тока вдоль поверхности обрабатываемой детали [5-7], приводятся математические модели взаимодействия пучка с поверхностью $[8,9]$, однако описания конкретного алгоритма по уменьшению ошибок формы поверхности, его возможностей по уменьшению амплитуды неоднородностей с различным латеральными размерами (пространственными частотами) не приводится. Ранее для решения данной задачи нами была разработана программа „MIMADD“, модели- 


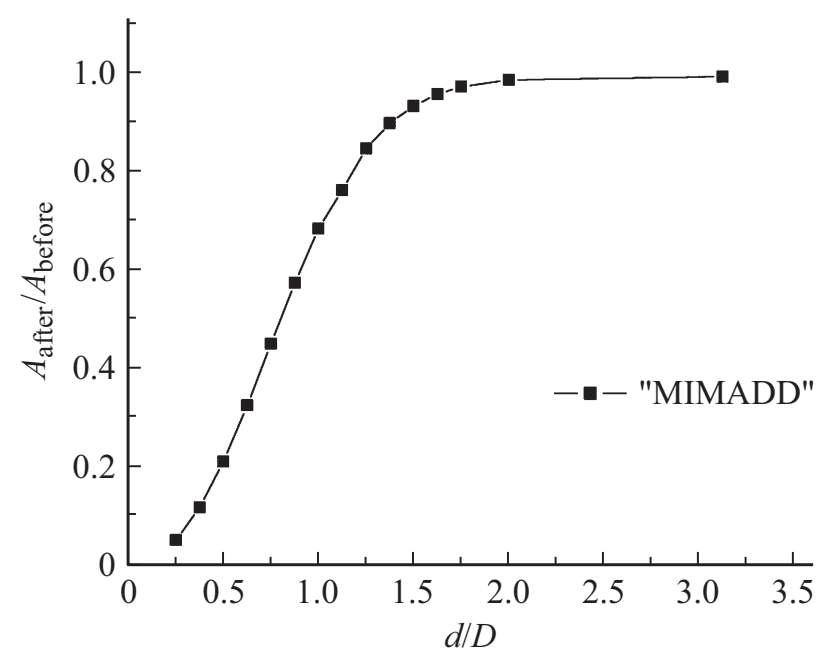

Рис. 1. Чувствительность метода к латеральному размеру неоднородности.

рующая взаимодействие ионного пучка заданной формы и размера с поверхностью [5].

\section{Локальная коррекция ошибок формы}

Для решения задачи коррекции локальных ошибок формы поверхности оптических элементов применяется источник ионов с малым по сравнению с диаметром обрабатываемой детали $(L)$ ионным пучком $(d)$, т.е. $d \ll L$. В процессе работы источника происходит физическое распыление поверхности за счет бомбардировки ускоренными ионами, образуется кратер травления. Характеристики кратера (профиль сечения, проходящего вдоль оси ионного пучка, ширина на полувысоте и глубина) зависят от параметров и времени работы источника. Назовем распределением скорости травления зависимость глубины кратера травления, полученного за единицу времени, от координат. При постоянстве внешних параметров (параметры ионного источника, расстояние от источника до поверхности, угол падения ионов на поверхность и др.) профиль травления, полученный за время $T$, будет пропорционален профилю скорости травления с коэффициентом пропорциональности $T$, т.е.

$$
s(\boldsymbol{\rho})=T \cdot V(\boldsymbol{\rho}),
$$

где $s(\boldsymbol{\rho})-$ профиль ионного пучка, $V(\boldsymbol{\rho})-$ скорость травления $[\mathrm{nm} / \mathrm{s}]$.

Введем еще одно понятие: карта поверхности дискретный набор координат и значений высоты рельефа в них $S(\boldsymbol{\rho})$, таким образом, $S(\boldsymbol{\rho})$ - исходная поверхность $[\mathrm{nm}]$. Карта поверхности представляет собой набор точек, соответствующих отклонению реальной высоты в данной точке от расчетной (идеальной) поверхности. Карта поверхности получается измерением формы поверхности интерферометрическими методами, например, на интерферометре с дифракционной волной сравнения [10]. Таким образом, задача коррекции формы поверхности может быть представлена как математическая задача: зная карту поверхности $S(\boldsymbol{\rho})$ и распределение скорости травления на пучке $V(\boldsymbol{\rho})$, необходимо построить карту времен травления $T(\boldsymbol{\rho})$ (время работы пучка в каждой координате поверхности), приводящей к уменьшению локальных ошибок формы (рис. 1).

\section{Анализ программы „MIMADD“}

В программе „MIMADD“ использована простая модель для времен травления:

$$
T\left(\rho_{i}\right)=\gamma S\left(\rho_{i}\right)
$$

т.е. время травления в точке равно амплитуде поверхности в данной точке, умноженной на некоторый коэффициент $\gamma$, где числовой параметр $\gamma$ нужно определить из условия минимума функционала $\Phi_{0}$, учитывающего воздействие пучка как на точку, в которой он находится, так и на соседние точки в пределах пятна распределения ионного тока (в задаче предусматривается шаг сканирования - расстояние между соседними точками карты поверхности step $\ll d)$ :

$$
\Phi_{n}=\left\|S(\boldsymbol{\rho})-\Sigma T_{i} V\left(\boldsymbol{\rho}-\boldsymbol{\rho}_{i}\right)\right\|^{2} .
$$

Модель прекрасно справляется с тестовыми поверхностями, уменьшая среднеквадратическое отклонение ошибок формы от „идеала“ до требуемого (согласно критерию Марешаля) значения. Тем не менее при работе с реальными поверхностями был обнаружен ряд недостатков используемого алгоритма.

1. В силу выбранной модели времен травления (4) результирующая поверхность повторяет исходную, но с уменьшением амплитуды неоднородностей, связанных с размером пучка следующей зависимостью (рис. 1). На рисунке представлена рассчитанная зависимость чувствительности модели к соотношению размера ионного пучка к латеральному размеру неровности на поверхности. Представлено как с ростом латерального размера неоднородности уменьшается ее амплитуда ( $A_{\text {before }}-$ амплитуда до травления, $A_{\mathrm{after}}-$ амплитуда после травления).

Таким образом, результатом работы алгоритма становится поверхность, на которой проявляются неоднородности более высоких пространственных частот (латеральный размер меньше размера пучка). А поскольку обработка поверхности подложки при ее производстве происходит при осевом вращении, на поверхности проявляется высокочастотная кольцевая структура. Эти кольца, являясь по своей сути, сферической аберрацией высоких порядков, приводят к размытию изображения, и, как следствие, снижению разрешающей способности оптической системы, даже если критерий Марешаля выполнен.

2. Скачек производной на границе (край зеркала и/или центральное отверстие) приводит к развитию рельефа 
и, как следствие, снижает эффективность реализации программного алгоритма по минимизации RMS. Это приводит к необходимости виньетирования оптических элементов и снижению светосилы системы.

В настоящей работе предложен другой подход к решению данной задачи — „итерационный“ метод улучшения поверхности.

\section{„Итерационный“ метод улучшения поверхности}

Концепция метода основана на поиске координаты на карте поверхности, на которой применение пучка за некоторое малое время $\tau$ приведет к наибольшему улучшению поверхности в сравнении с другими точками. После проведения поиска осуществляется „травление“ в найденном оптимальном положении пучка, вследствие этого поверхность меняется, и на ней также ищется наилучшая позиция. Если на поверхности не существует такого места, работа в котором за время $\tau$ улучшила бы выбранный критерий, производится уменьшение времени травления в точке, т.е. $\tau_{i+1}=\tau_{i} / 2$. Таким образом, многократное повторение данного алгоритма должно приводить к улучшению поверхности.

Рассмотрим подробнее выбор критерия, по которому будет оцениваться изменение поверхности. В основе построения задачи лежит критерий Марешаля, исходя из которого необходимо следить за динамикой RMS-поверхности на каждой итерации алгоритма.

$$
\mathrm{RMS}=\sqrt{\frac{1}{N} \Sigma_{\rho}(S(\boldsymbol{\rho})-\bar{S})^{2}},
$$

где $N$ - количество точек на карте поверхности, $\bar{S}-$ среднее арифметическое по всей поверхности.

Однако для вычисления RMS необходимо учесть все значения поверхности. Это означает, что сложность однократного нахождения RMS порядка $O(N)$ (оценочная сложность алгоритма [11]), где $N-$ количество

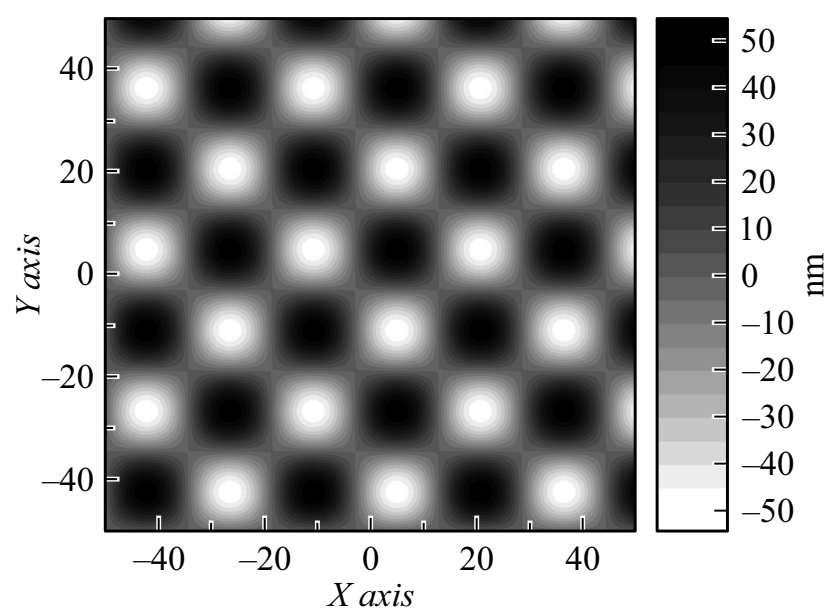

Рис. 2. Тестовая поверхность (размерность $200 \times 200$ точек). Чередование ям и бугров с периодом 60 точек. $P-V=100 \mathrm{~nm}$, $\mathrm{RMS}=24.5 \mathrm{~nm}$.

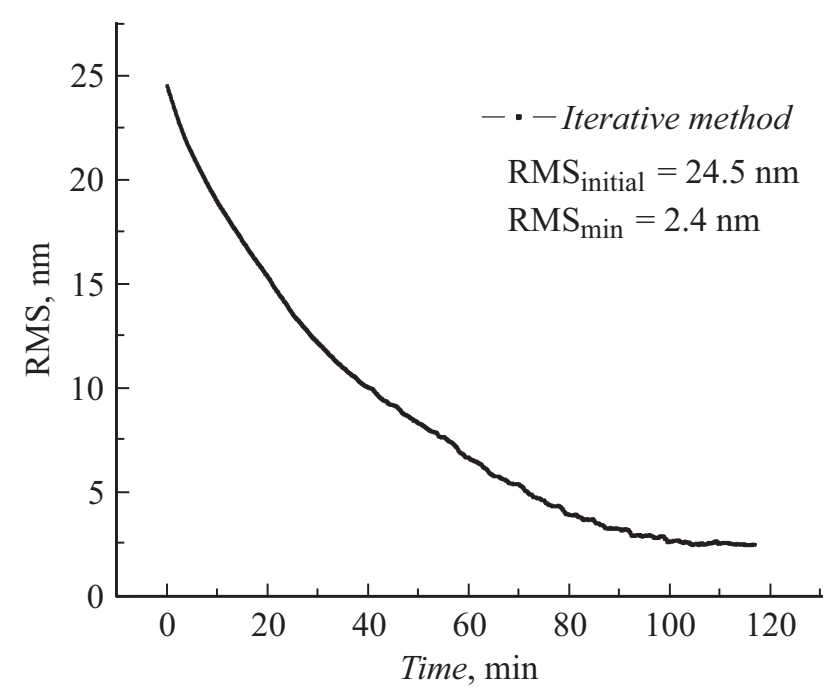

Рис. 3. Расчетная зависимость спадания RMS от времени травления (расчет осуществлен за 200000 итераций).

точек поверхности, а сложность всего метода становится $O\left(K^{*} N^{2}\right)$, где $K-$ количество итераций. Тестовая поверхность, для которой проводились вычисления в настоящей работе (рис. 2), задана с разрешением $200 \times 200$ точек, что дает $N=40000$.

Таким образом, даже однократный расчет оптимальной точки занимает порядка $0.01 \mathrm{~s}$, в то время как возможность следить за другой характеристикой поверхности, улучшение которой так же будет приводить к снижению RMS, может обеспечить меньшее время расчета. Для уменьшения времени расчета необходимо, чтобы критерий проверялся по меньшему количеству точек. Оптимальной является проверка на пятне травления, так как поверхность меняется лишь на нем. Поскольку RMS не является аддитивной характеристикой, уменьшение RMS на пятне травления не позволяет сделать вывод об уменьшении RMS всей поверхности. Поэтому был выбран другой критерий, вычисление которого занимает меньшее время, а его уменьшение так же ведет к снижению RMS (рис. 3), а именно уменьшение суммы модулей производных на пятне травления (7).

Использование данного критерия приводит к тому, что после травления суммарный наклон поверхности в месте травления уменьшается:

$$
\Sigma_{\rho}^{R}\left|S(\rho)_{x}^{\prime}\right|+\left|S(\rho)_{y}^{\prime}\right|
$$

Сложность всего метода порядка $O\left(K^{*} N^{*} n\right)$, где $K-$ количество итераций, $N-$ количество точек карты поверхности, $n-$ количество точек на пятне травления. Таким образом, удалось уменьшить сложность метода (время расчета) в $N / n$ раз.

\section{Результаты и обсуждения}

Для оценки работоспособности метода были проведены сравнительные расчеты обработки тестовой поверх- 

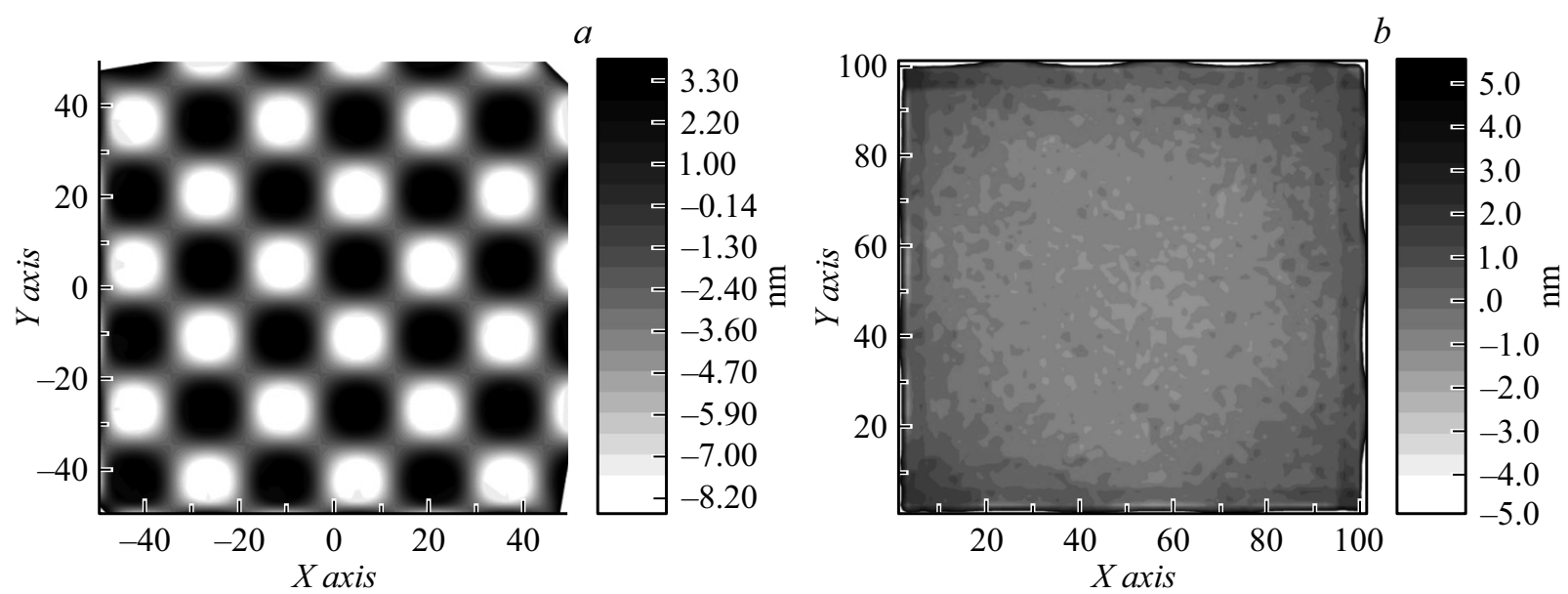

Рис. 4. Результат обработки тестовой поверхности „ионным пучком“ $d / D=0.25: a-$ программа „MIMADD“, $b-$ итерационный алгоритм.

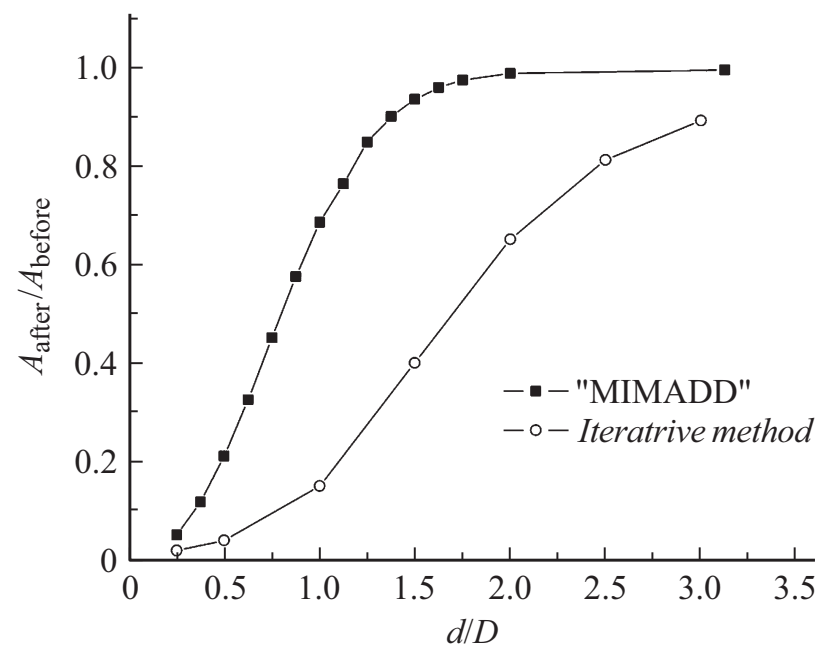

Рис. 5. Чувствительность метода к латеральному размеру неоднородности.

Результаты моделирования

\begin{tabular}{l|c|c|c}
\hline$d / D$ & Время, $\mathrm{s}$ & RMS $_{\text {МiмADD, }} \mathrm{nm}$ & RMS $_{\text {iterat. method, }} \mathrm{nm}$ \\
\hline 1 & 1611 & 14.43 & 3.18 \\
0.5 & 6173 & 4.44 & 0.63 \\
0.25 & 23372 & 1.16 & 0.6
\end{tabular}

ности (рис. 2) „ионными пучками“ различного размера. Рассматривались „пучки“ размером $d=1,0.5$ и 0.25 относительно размера неоднородности $(D)$ тестовой поверхности. Результаты моделирования приведены в таблице. Примеры результирующих поверхностей представлены на рис. 4.

Как можно видеть, для всех рассмотренных размеров пучка результирующая ошибка поверхности по параметpy RMS, полученная при реализации итерационного алгоритма, заметно превосходит результаты, полученные с помощью программы „MIMADD“. Кроме того, как и для программы „MIMADD“, была проведена оценка чувствительности метода к латеральному размеру неоднородности, т.е. рассматривалось, как пучок некоторого размера $d$ уменьшает амплитуду неоднородности латерального размера $D$. Результаты моделирования в сравнении с результатами, полученными в программе „MIMADD“, представлены на рис. 5.

Как можно видеть, итерационный метод позволяет значительно продвинуться в область более высоких пространственных частот. Если программа „MIMADD“ успешно справляется (амплитуда уменьшается вдвое) с неоднородностями размером порядка ионного пучка, в то время как итерационный метод позволяет снижать амплитуду неоднородностей латерального размера вдвое меньше размера ионного пучка. Это позволит уменьшить как количество итераций при коррекции формы поверхности, поскольку можно воздействовать на больший диапазон пространственных частот без уменьшения размера пучка, так и время обработки поверхности. Поскольку уменьшение размера пучка, производится за счет диафрагмирования $[8,12]$, а это приводит к снижению тока ионного пучка, а как следствие, уменьшению объема удаляемого материала квадратично к диаметру диафрагмы.

На рис. 6 представлена обработка реальной карты поверхности (полученной на интерферометре с дифракционной волной сравнения [10]) программой „MIMADD“ и итерационным алгоритмом. Как и в случае с тестовой поверхностью, предложенный метод показал заметно более высокое качество поверхности по сравнению с программой „MIMADD“. Важным результатом является заметно меньшее проявление кольцевой структуры ошибок формы поверхности, являющейся сферической аберрацией высокого порядка. Что должно привести к повышению качества изображения (повышения разрешающей способности оптической системы). Однако наблюдаются кольца на внешнем и внутреннем контуре поверхности, сформировавшиеся из-за скачка производной на границе. 

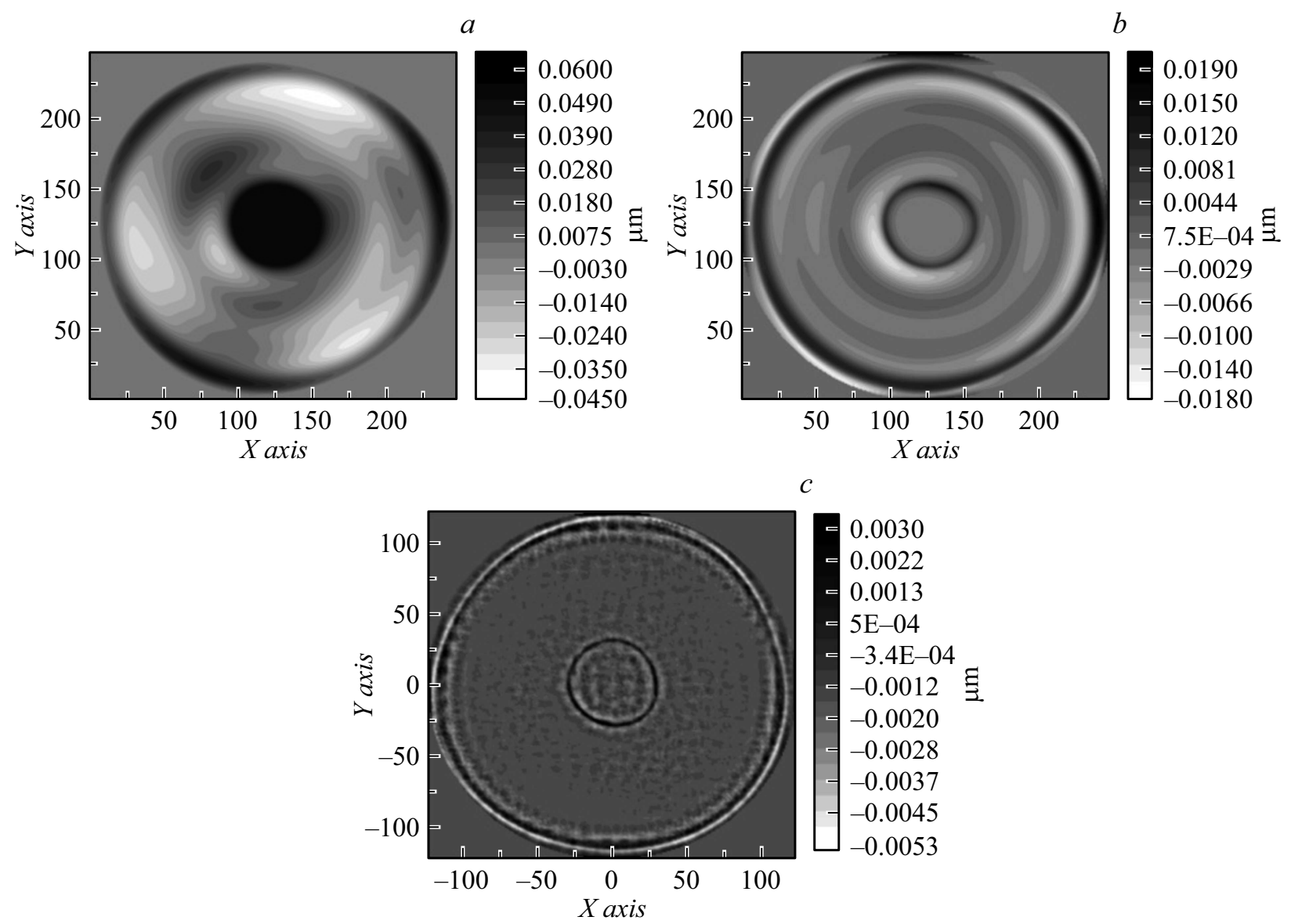

Рис. 6. Сравнение результатов работы двух алгоритмов для реальной поверхности: $a-$ исходная поверхность $(P V=105 \mathrm{mn}$, $\left.\mathrm{RMS}=18.7 \mathrm{~nm}, \mathrm{RMS}_{\text {angle }}=0.32 \mathrm{mrad}\right) ; b-$ результат работы „MIMADD“ $\left(P V=37.0 \mathrm{~nm}, \mathrm{RMS}=4.4 \mathrm{~nm}, \mathrm{RMS}_{\text {angle }}=0.19 \mathrm{mrad}\right)$; $c$ - результат работы „итерационного“ метода $\left(P V=8.3 \mathrm{mn}, \mathrm{RMS}=3.06 \mathrm{mn}, \mathrm{RMS}_{\text {angle }}=0.14 \mathrm{mrad}\right)$.

Для решения данной проблемы в программу добавлена возможность виртуального расширения области травления в сторону наружного и внутреннего контура, без скачка производной, на величину пучка. В результате кольцевая структура будет образовываться на виртуальном крае поверхности (за габаритами детали), на реальном краю поверхности развития рельефа происходить не будет. Это позволит сохранить реальные габариты поверхности (не прибегать к виньетированию).

\section{Выводы}

Реализация предложенного в работе алгоритма требует значительно больше машинного времени (расчет одной карты занимает время порядка $1 \mathrm{~h}$ ), чем поиск локального минимума функционала $\Phi_{0}$ с помощью генетического алгоритма (порядка минуты), реализованного в программе „MIMADD“. Однако результат работы „итерационного алгоритма“ значительно превосходит результат, получаемый с помощью „MIMADD“, как по параметру RMS, так и по форме результирующей поверхности. На результирующей поверхности, рассчитанной по „итерационному алгоритму“, значитель- но меньше проявляется детерминированная кольцевая структура, приводящая к аберрации изображения и, как следствие, снижению разрешающей способности оптической системы. Расширение функционала программы за счет виртуального расширения карты поверхности без скачка производной во внешний и внутренний контуры позволят избежать развития рельефа на краях заготовки, следовательно, сохранить реальные габариты поверхности (позволит не прибегать к виньетированию).

\section{Финансирование работы}

Работа выполнена при поддержке Российского фонда фундаментальных исследований: 18-32-00149мол_а, 18-07-00633 и программы Российской академии наук 0035-2018-0018.

\section{Конфликт интересов}

Авторы заявляют, что у них нет конфликта интересов. 


\section{Список литературы}

[1] Малышев И.В., Пестов А.Е., Полковников В.Н., Салащенко Н.Н., Торопов М.Н., Чхало Н.И. // Поверхность. Рентгеновские, синхротронные и нейтронные исследования. 2019. № 1. С. 1-11.

[2] Born M., Wolf E. // Principles of Optics (Cambridge University). 1999. Sec. 9.3. P. 528.

[3] Arnold T., Bohm G., Fechner R., Meister J., Nickel A., Frost F., Hansel T., Schindler A. // Nucl. Instrum. Methods Phys. Res. Sect. 2010. A 616. P. 147-156.

[4] Jiao C., Li S., Xie X., Chen S., Wu D., Kang N. // Appl. Opt. 2010. Vol. 49. N 4. P. 578-585.

[5] Chkhalo N.I., Kaskov I.A., Malyshev I.V., Mikhaylenko M.S., Pestov A.E., Polkovnikov V.N., Salashchenko N.N., Toropov M.N., Zabrodin I.G. // Precision Engineering. 2017. Vol. 48. P. 338-346.

[6] Schindler A. Optical Fabrication and Testing. 2012. Monterey, California United States 24-28 June 2012. P. OW4D.1.

[7] Schmelzer O., Feldkamp R. // Proc. SPIE. 2015. Vol. 9633. P. $96330 \mathrm{E}$.

[8] Liao W., Dai Y., Xie X., Zhou L. // Appl. Opt. 2014. Vol. 53. N 19. P. 4266-4274.

[9] Liao W., Dai Y., Xie X., Zhou L. // Appl. Opt. 2014. Vol. 53. N 19. P. 4275-4281.

[10] Chkhalo N.I., Malyshev I.V., Pestov A.E., Polkovnikov V.N., Salashchenko N.N., Toropov M.N., Soloviev A.A. // Appl. Opt. 2016. Vol. 55. N 3. P. 619-25.

[11] Разборов А.А. // Математическое просвещение. МЦНМО. 1999. № 3. C. 127-141.

[12] Электронный ресурс. Режим доступа: http://www.opteg.com 